Pathologe 2021 - 42 (Suppl 2):S207-S209 https://doi.org/10.1007/s00292-021-00989-8 Angenommen: 3. September 2021 Online publiziert: 5. Oktober 2021 (c) Springer Medizin Verlag $\mathrm{GmbH}$, ein Teil von Springer Nature 2021

\section{Karl Rudolf Caesar}

\author{
$14.03 .1929-11.01 .2021$
}

K. Donhuijsen
Braunschweig, Deutschland

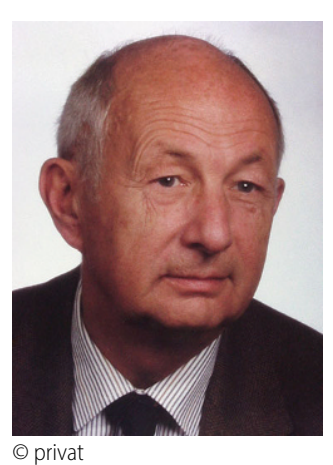

Am 11. Januar 2021 verstarb Herr Professor Dr. med. Karl Rudolf Caesar im Alter von 91 Jahren. Sein letzter Gruß, abgestempelt am 10.01.2021, an den Unterzeichner lautet: „... und bewahren Sie sich Ihre Zuversicht". Er starb (klinisch gesehen) unvermittelt an den Folgen einer ausgedehnten Hirnembolie bei bekanntem und vor längerer Zeit operativ behandeltem Mitralklappenvitium. Mit diesem Ereignis hatte er gerechnet.

Unsere erste Begegnung erfolgte anlässlich meiner Bewerbung zu seiner Nachfolge als Chefarzt des Pathologischen Institutes am Städtischen Klinikum Braunschweig Ende 1990. Bei der Führung durch sein vorbildlich geordnetes Institut war offenkundig: Er war der respektierte Chef, die fachliche Autorität und zugleich eine väterliche Instanz für die etwa 30 Mitarbeiterinnen und Mitarbeiter, deren Geburtstage er auswendig kannte. In seiner zurückhaltenden, nüchternen Art waren ihm Chefarztallüren fremd, auch wenn seine Generation Freiheiten und kleine Privilegien (Autopflege im Institut) hatte, die heute undenkbar sind. Sein Arbeitsraum war hell und sachlich, sein Besprechungsraum eine umfangreiche, überwiegend englischsprachige Bibliothek in starkem Dämmerlicht. Seine Informationen an mich waren knapp und neutral gehalten. Erst nach der Wahl im ehrwürdigen großen Sitzungssaal des neugotischen Rathauses der alten Hansestadt erfolgten ausführliche, wertende Mitteilungen zu den Kliniken und ihren Chefärzten, zur Institutsstruktur und seinen Mitarbeitern, zu einsendenden Kollegen und zur zuständigen Alma mater und ihren Lehraufträgen. Am Abend des 30.04.1991 erfolgte bei einem Glas Sekt im Labor die Schlüsselübergabe zum frisch gestrichenen Institut. Der Abschied von der geliebten Arbeit fiel Caesar sichtlich schwer. Der hervorragende Ruf des Institutes war ein Ergebnis seines über 20jährigen Einsatzes für eine evidenzbasierte Medizin, für möglichst objektivierbare diagnostische Parameter, für eine kritischwissenschaftliche Methodik, getragen von seiner selbstreflexiven, kognitiven Distanz. Er betrachtete sich in erster Linie als $\mathrm{Na}$ turwissenschaftler und Mediziner und erst dann als Arzt für Pathologie.

Sein Weg in das Braunschweiger Institut war nicht vorhersehbar.

Karl Rudolf Caesar wurde am 14.03.1929 geboren - in den Iden des Märzes. Ob bei dieser besonderen Konstellation der einzige Sohn in apotropäischer Absicht die Vornamen von gleich zwei großen Kaisern erhielt, können wir nur vermuten. Der Vater war als selbstständiger Pharmazeut indirekt an der Pharmafabrik Caesar \& Loretz in Halle an der Saale (heute "Caelo" in Hilden/Rheinland) beteiligt. Sein großes Wissen über Arzneipflanzen gab er später noch gern und anschaulich an seine Enkel weiter. Einer der Urgroßväter mit Namen Caesar war Apotheker in Katzenelnbogen im Rhein-Lahn-Kreis, Rheinland-Pfalz. Eine vier Jahre ältere Schwester lebt bis heute in Haldensleben. Mit dem 16. Lebensjahr wurde Caesar noch im März 1945 „zur Verteidigung der Alpenfestung" als Sol- 
dat eingesetzt. Nach dem Abitur in Halle nahm er 1947 das Medizinstudium in Kiel auf, das er 1954, nach zwischenzeitlichem Semester in Freiburg, mit der Note "sehr gut" abschloss. Bei dem Kieler Anatom W. Bargmann promovierte Caesar 1954 mit einer histochemischen Arbeit "Zur Zytologie der Inselorgane von Teleostiern mit besonderer Berücksichtigung des Kolloidvorkommens". Das zur Untersuchung erforderliche Pankreasgewebe verschiedener Fischarten asservierte er selbst fangfrisch auf Fischkuttern im Nordatlantik. Anfang der 1950er-Jahre setzte dieser Einsatz zweifellos erhebliche Nehmerqualitäten voraus. Eine Abmusterungsbescheinigung von dieser Tätigkeit als „Hilfsmatrose" widerlegt jeden Verdacht auf Seemannsgarn.

Im Kieler Institut für Anatomie lernte er seine zukünftige Ehefrau Elke, geb. Schröder, kennen. Prof. Bargmann empfahl Caesar an Prof. Hans Ruska (1908-1973), den medizinischen Anwender des von seinem Bruder Ernst entwickelten Elektronenmikroskopes (Physiknobelpreis 1986), der damals in den USA tätig war. Aus der über einjährigen Arbeit im Forschungslabor des State Dep. of Health, Albany, New York, gingen mehrere hochrangige Publikationen mit H. Ruska und G. A. Edwards hervor. Von dort ging Caesar auf Empfehlung von Ruska nach Tübingen zu dem Ordinarius für Pathologie Erich Letterer (1895-1982). Hier baute er maßgeblich die elektronenmikroskopische Abteilung auf und bearbeitete allgemeinpathologische Themen. Es entstanden weitere Publikationen zur Ultrastruktur von Nerven, zum Reizleitungssystem des Herzmuskels, zur Leberzellmembran, zur Proteinproduktion in Plasmazellen, zur Immunkomplexbildung in Glomerula u. v. a. m. Caesar beschrieb als erster in Europa für die damals noch getrennten Amyloidarten eine einheitliche fibrilläre Ultrastruktur, zeitgleich mit dem US-Amerikaner Evan Calkins. Die Jahre in Tübingen von 1958 bis 1964 waren retrospektiv seine interessanteste und wissenschaftlich ertragreichste Zeit. Seine Korrespondenz aus diesen Jahren, in zwei Aktenordnern erhalten, belegt vielfältige fachliche Kontakte mit Kollegen verschiedenster Fachrichtungen in mehreren Kontinenten und zahlreichen europäischen Instituten, abgefasst in perfektem
Englisch und geschliffenem Französisch. Seine Methode, die Elektronenmikroskopie, war der Hype und die wissenschaftliche Hoffnung dieser Zeit. So wurden zahlreiche neue Spezialabteilungen gegründet mit erheblichen Geräteinvestitionen selbst in nichtuniversitären Instituten. Die Qualität der von Caesar gelieferten Abbildungen zur Ultrastruktur war für viele Kollegen der anzustrebende Maßstab. Seit 1960 erhielt Caesar immer wieder Stellenangebote von universitären und industriellen Einrichtungen u.a. von $\mathrm{H}$. Ruska, jetzt in Düsseldorf und - hochdotiert - von Henry Rappaport, AFIP, Washington. Er blieb in Tübingen, da ihm das Gebiet der UItrastruktur allein zu schmal war und sich die Familie vergrößert hatte. Caesar habilitierte 1963 zu dem Thema „Prinzipien des morphischen Ablaufes einer Zellschädigung an verschiedenen Zelltypen. Eine Untersuchung mit Hilfe der Elektronenmikroskopie." Seine Antrittsvorlesung am 23.06.1964 trug das Thema: „Allgemeine Cytopathologie der Leberzelle“. Über die DFG konnte er für seine Forschungen in Tübingen Anfang der 1960er-Jahre Drittmittel in fünfstelliger Höhe mobilisieren.

Mit dem bevorstehenden Ausscheiden von E. Letterer wurden 1964 Wechselgedanken drängend. Die Rückkehr von W. Masshoff, Berlin, nach Tübingen drohte und in dieser Situation folgte Caesar einer Anfrage Karl Lennerts, vorrangig aus familiären Gründen, weil in Kiel seine Schwiegereltern lebten und die Jahre bei Bargmann positiv nachklangen. Schriftlichen und mündlichen Warnsignalen und einem Angebot vom neuen Direktor Bohle in Tübingen meinte er nicht folgen zu können, da er bei Lennert im Wort war. Am 15.09.1964 begann Caesar am Kieler Institut. Erwartungsgemäß ging es schief. Die Briefe zum Jahreswechsel an Kollegen beklagen den Fehler eines Wechsels nach Kiel; fachlich und auch sonst sei alles „viel zu kleines Karo. - Ob ich hier nochmal wegkomme?" Das hohe Primat der Lymphomklassifikation in Kiel hatte Caesar, der an den allgemeinen Prinzipien der Pathologie und nicht an Kategorisierungen interessiert war, wohl falsch eingeschätzt. Lennert dagegen hatte die Brauchbarkeit der Ultrastruktur für seine Zwecke überbewertet. Zudem war die Kluft zwischen dem aktiven Alt-Ka- tholiken und dem naturwissenschaftlich geprägten "Generalisten“ zu tief. Caesar ging in die innere Emigration, lebte von der wissenschaftlichen Substanz, schrieb mehrere Lehrbuch- und Handbuchartikel zu Ultrastruktur und Herzpathologie und vergrub sich in die Routinearbeit. Seine Antrittsvorlesung in Kiel erfolgte im November 1965 über „Entwicklungslinien der Amyloidforschung". Anregungen von Kollegen, sich auf einen Lehrstuhl zu bewerben - z. B. Ulm -, konterte er: für ein solches Vorhaben sei er zu leise. Bemerkenswerterweise nahm er bereits 1965 erstmals an der "Rätselecke" in Hannover teil. Bei diesen praxisorientierten Pathologentagungen Norddeutschlands verblüffte Caesar bis zum Jahr 2000 immer wieder mit seiner hohen Fachkenntnis.

Zu Silvester 1967 wurde dem inzwischen dreifachen Vater (eine Tochter, 2 Söhne) von Kollegen zum bevorstehenden Wechsel nach Braunschweig gratuliert. Dort war er zunächst kommissarischer Leiter während einer Krankheitsphase seines dortigen Vorgängers und ab 1969 mit 40 Jahren Chefarzt des ehemals renommierten Institutes am Städtischen Klinikum, einem 1500-Betten-Krankenhaus. Das Institut sieht seine Wurzeln im Herzoglichen Anatomisch-Chirurgischen Institut der Carolina von 1750, einer Vorläufereinrichtung der heutigen Technischen Universität Braunschweig. Im Februar 1970 wurde Caesar zum außerplanmäßigen Professor für Pathologie an der Universität Kiel ernannt. Sein Braunschweiger Institut versorgte alle Arztpraxen und alle Kreiskrankenhäuser der gesamten Region. Mit einfachen, aber wirksamen Mitteln verbesserte er die Qualität des Gewebelabors. Sein Vorgänger war überwiegend biochemischer Laborarzt, in zweiter Linie leidenschaftlicher Jäger und an dritter Stelle Pathologe. Caesar brachte die mikroskopische Diagnostik rasch auf amerikanisches Niveau. Er forderte die jungen Kollegen und Durchgangsassistenten in angemessener Weise und blieb innen oft später noch als Mentor verbunden. Caesar engagierte sich für die Braunschweiger niedergelassenen Ärzte und ihre Patienten, immer klug ausgleichend und als Ratgeber im Hintergrund. Über lange Jahre war er in Klinikleitung, Ärztekammer und Ärzteverein tätig, auch 
hier nie als „Frontman“. Seine zahllosen Fortbildungsvorträge zeugten von der traditionellen Bedeutung und diagnostischen Breite unseres Faches. Im Jahr 1982 erhielt er die Ernst-von-BergmannPlakette, die höchste Auszeichnung der Bundesärztekammer für sein Wirken in der kollegialen Fort- und Weiterbildung.

Caesar war ein Ästhet. Obduktionsprozedere, histologische Analysen und Befundberichte waren formvollendet. Sein neues Architektenhaus aus den 1970erJahren, am Stadtrand zur Heide hin gelegen, ist auch heute noch ein Musterbeispiel der klassischen Moderne. Es ist stilsicher und geschmackvoll eingerichtet und bewahrt seitdem eine besondere kunstsinnige Aura, etwa mit Bildern des befreundeten Pathologen und Malers Friedrich Lindlar (1924-2012), Berlin. Caesars musikalische Vorliebe gehörte Georg Friedrich Händel, wohl geprägt durch seine Herkunft aus der Händel-Stadt Halle.

Caesar war ein feinfühliger Analytiker mit fundiertem naturwissenschaftlichem Denken. Zur Dokumentation der makroskopischen Befunde, besonders für seine Buchbeiträge zur Pathologie des Herzens, benutzte er hochwertige Mittelformatkameras aus Braunschweiger Produktion (Rollei). Die Freude an neuen Erkenntnissen hielt inn beweglich und geistig frisch bis zuletzt. Fachlich setzte er im Braunschweiger Institut seit den 1980erJahren die immunhistochemische Methode ein. Später begleitete er die molekularpathologische Einrichtung des Institutes mit aktivem Interesse. In Braunschweig brachte er einige Promotionen zum Abschluss. Die gute Beziehung zu seinen Kieler Kollegen (und nicht nur diesen) blieb lebenslang erhalten, besonders zu seinem Kieler Mitoberarzt Wolfgang Remmele und zu seinem Doktoranden Konrad MüllerHermelink. Von Karl Lennert hing eine Fotografie mit Widmung in seinem Arbeitszimmer.

Caesar war ein Meister einer differenzierten Argumentation, vorgetragen mit einem feinen Florett, dessen Stich der Kontrahent zunächst kaum wahrnahm. Dem schweren Säbel dagegen wich er in der fachlichen Konfrontation geschickt aus. Eingeladen in eine Expertenrunde zur Kassenärztlichen Bundesvereinigung KBV nach Köln Anfang der 1990er-Jahre, ver- hinderte Caesar mit kluger Argumentation die beabsichtigte Einführung einer Entlohnung ausschließlich für positiv gestellte, verschlüsselbare histologische Diagnosen. Der Pathologe als Ausschlussdiagnostiker oder Differenzialdiagnostiker war der KBV bis dahin nicht bekannt. Die Umsetzung des SGB V Ende der 1980er-Jahre, mit dem faktischen Verbot von Klinikärzten für KVKollegen zu arbeiten, betraf auch Braunschweig und wurde hier sehr rigide von den örtlichen KV-Funktionären umgesetzt; den Kollegen, die Caesar immer außerordentlich engagiert unterstützt hatte. Seine Gegenargumente wie etwa eine stark reduzierte Weiterbildungsmöglichkeit für angehende Fachärzte verfingen nicht. Heutige Probleme wie Nachwuchsmangel und fehlende Breite in der Weiterbildung sah er voraus. In dieser Situation reichte der städtische Beamte Caesar mit 62 Jahren seinen vorzeitigen Ruhestand ein ein Fehler, wie er später zugab.

Caesar war ein Pragmatiker und wenn nötig auch ein Kämpfer, zumeist allerdings im Stillen und nie gegen Windmühlenflügel. Die von den chirurgischen Kollegen entnommenen Menisci untersuchte er nur, wenn er vollständige klinische Angaben erhielt. Andernfalls hob er sie nur auf. Mit Plazenten verfuhr er ähnlich. Caesar hatte keine Feinde und mied oberflächliche Freundschaften, etwa im gesellschaftlichen Rahmen. Ein Zucken des Mundwinkels war sein höchster Grad einer Missbilligung. Caesars zurückhaltende, selbstlose Art, die immer verbunden war mit einer positiven und stärkenden Sicht seines Gegenübers, führte zu einer breiten Wertschätzung und seinem besonderen Nimbus. Prosaischer ging es dann beim Hochschulsport an der Technischen Universität Braunschweig zu. Hier spielte er nicht ohne Ehrgeiz mit der ganzen Familie Tennis. Einem unangemeldetem Interview hierzu mit Journalisten eines Managermagazins konnte er nicht ausweichen und erfreute sich dann doch an dem gelungenen Artikel. Auch beim Radeln zwischen Harz und Heide entwickelte er Ambitionen, wie der Berichterstatter weiß. Im Ruhestand verlegte er die Laborarbeit in die heimische Küche und kochte erfolgreich, z.B. Hechtklößchensuppe. Nun wurde endlich auch der Garten selbst gepflegt.
Caesar war ein Familienmensch und ein sensibler Freund. Neben dem fordernden Beruf stand die Familie im Mittelpunkt, wenn auch Elke Caesar lange die Hauptlast trug. Eltern und Schwiegereltern wurden lange umkümmert. Die Kinder wurden groß und gingen erfolgreich ihren Weg: Sophie Verlagslektorin in Spanien, Helmuth Architekt, Friedrich Neurologe. Elke Caesar fand Zeit für ihre kunsthistorischen und literarischen Interessen und widmete sich aktiv der Buchbindekunst. Mit der Pensionierung Caesars erhielt die Familie mehr Zuwendung. Das Anwesen in Mallorca wurde nun häufiger besucht, besonders in trüben Zeiten. Caesar berichtete gern über neue Ergebnisse zur Familienforschung, die er noch bis vor den Dreißigjährigen Krieg ausweiten konnte. Er korrespondierte regelmäßig mit seinen Kindern und später den Enkeln, gelegentlich auch mit uns zu ausgewählten Gedichten. Die Beschwerlichkeiten des Alters nahm er achselzuckend hin. Die diamantene Hochzeit wurde vor einigen Jahren still begangen. Unsere regelmäßigen Besuche in seinem Heim waren immer bereichernd und den geistvollen Gesprächen und (literarischen) Anregungen bis in die jüngste Zeit, verdanken wir viel. Caesars begleiteten unsere Kinder bei Abitur und Hochzeit. Seine Bonmots "die Protestanten suchen's und die Katholiken haben's!" sowie seine Frage „Wie schlafen denn die Fische?" wurden bei uns sprichwörtlich. Seine anhaltende Verbundenheit zum Fach, zum Institut und zu seinen Mitarbeitern (Weihnachtsfeiern inklusive) übertrug sich besonders auch auf seinen derzeitigen Nachfolger im Amt, den er wegen seiner hohen Qualitäten außerordentlich schätzte.

Die DGP wurde 1887 in Braunschweig gegründet. Karl Rudolf Caesar war Mitglied vom 04.03.1961 bis zu seinem Tod am 11.01.2021, also über fast 60 Jahre.

The supplement containing this article is not sponsored by industry.

\section{Korrespondenzadresse}

\section{Prof. Dr. K. Donhuijsen}

Helgolandstr. 55b, 38108 Braunschweig, Deutschland

donhuijsen@t-online.de 